\title{
Exchange and correlation near the nucleus in density functional theory
}

\author{
Zhixin Qian \\ Department of Physics, Peking University, Beijing 100871, China
}

(Dated: May 6, 2018)

\begin{abstract}
The near nucleus behavior of the exchange-correlation potential $v_{x c}(\mathbf{r})$ in Hohenberg-Kohn-Sham density functional theory is investigated. It is shown that near the nucleus the linear term of $O(r)$ of the spherically averaged exchange-correlation potential $\bar{v}_{x c}(r)$ is nonzero, and that it arises purely from the difference between the kinetic energy density at the nucleus of the interacting system and the noninteracting Kohn-Sham system. An analytical expression for the linear term is derived. Similar results for the exchange $v_{x}(\mathbf{r})$ and correlation $v_{c}(\mathbf{r})$ potentials are also obtained separately. It is further pointed out that the linear term in $v_{x c}(\mathbf{r})$ arising mainly from $v_{c}(\mathbf{r})$ is rather small, and $v_{x c}(\mathbf{r})$ therefore has a nearly quadratic structure near the nucleus. Implications of the results for the construction of the Kohn-Sham system are discussed with examples.
\end{abstract}

PACS numbers: 71.15.Mb, 31.15.Ew, 71.10.-w 
Hohenberg-Kohn-Sham density functional theory (HKS-DFT) [1] is now possibly the most widely used tool for determining the electronic structure of atoms, molecules, and solids [2, 3]. In the implementations of HKS-DFT, the so-called Kohn-Sham (KS) noninteracting system which has electron density $\rho(\mathbf{r})$ equivalent to that of the interacting system is assumed. For the ground state of an (interacting) $N$-electron system in an external potential $v_{\text {ext }}(\mathbf{r})$, the one-particle orbitals $\phi_{n}(\mathbf{r})(n=1, \ldots N)$ of the corresponding KS system satisfy the KS equation

$$
\left[-\nabla^{2} / 2+v_{e x t}(\mathbf{r})+v_{H}(\mathbf{r})+v_{x c}(\mathbf{r})\right] \phi_{n}(\mathbf{r})=\epsilon_{n} \phi_{n}(\mathbf{r})
$$

with $\epsilon_{n}$ the eigenvalue. In Eq. (1),$v_{H}(\mathbf{r})$ is the classical Hartree potential, $v_{H}(\mathbf{r})=$ $\int d \mathbf{r}^{\prime} \rho\left(\mathbf{r}^{\prime}\right) /\left|\mathbf{r}-\mathbf{r}^{\prime}\right|$, and $v_{x c}(\mathbf{r})$ is the $\mathrm{KS}$ exchange-correlation (xc) potential which is the derivative of the KS exchange-correlation energy functional $E_{x c}^{K S}[\rho]$ with respect to the density $\rho(\mathbf{r}), v_{x c}(\mathbf{r})=\delta E_{x c}^{K S}[\rho] / \delta \rho(\mathbf{r})[1,2,3]$. The KS $x c$ potential takes account of all the correlations in HKS-DFT. These correlations arise from the Pauli exclusion principle, Coulomb repulsion, and correlation-kinetic effects. The former two effects are usually characterized with the Fermi-Coulomb hole [4], and the latter arises from the difference between the kinetic energy density of the interacting and the $\mathrm{KS}$ systems. $E_{x c}^{K S}[\rho]$ is usually separated into two parts, the exchange $E_{x}^{K S}[\rho]$ and correlation $E_{c}^{K S}[\rho]$ energy functionals. Correspondingly, $v_{x c}(\mathbf{r})$ is split into two components, the KS exchange $v_{x}(\mathbf{r})$ and correlation $v_{c}(\mathbf{r})$ potentials [1, 2, 3].

In practice, an approximation has to be made for the unknown $E_{x c}^{K S}[\rho]$. In this respect knowledge of the exact properties of $v_{x c}(\mathbf{r})$ is rather valuable to the construction of the KS system. In this paper it is shown that near nucleus,

$$
\bar{v}_{x c}(r)=\bar{v}_{x c}(0)+\frac{4}{3} Z\left[\bar{t}(0)-\bar{t}_{s}(0)\right] r / \rho(0)+O\left(r^{2}\right) .
$$

We use $\bar{f}(r)$ to denote the spherical average of a function $f(\mathbf{r})$. Here $Z$ is the charge of the nucleus, and $t(\mathbf{r})$ and $t_{s}(\mathbf{r})$ are the kinetic energy densities for the interacting system and the KS system, respectively. It is further shown that

$$
\bar{v}_{x, c}(r)=\bar{v}_{x, c}(0)+\frac{4}{3} Z \bar{t}_{x, c}(0) r / \rho(0)+O\left(r^{2}\right) .
$$

(By the notation $\bar{v}_{x, c}(r)$ we mean $\bar{v}_{x}(r)$ and $\bar{v}_{c}(r)$ respectively.) Here $t_{x}(\mathbf{r})$ is the first order correction to $t_{s}(\mathbf{r})$ in the adiabatic coupling constant perturbation scheme, (see later discussion and Ref. [5],) and $t_{c}(\mathbf{r})=t_{x c}(\mathbf{r})-t_{x}(\mathbf{r})$. Here $t_{x c}(\mathbf{r})$ is defined to be $t(\mathbf{r})-t_{s}(\mathbf{r})$. The 
result in Eqs. (22) and (3) is valid in general whether the system is an atom, molecule or a solid.

It is well known that, in the classically forbidden region of finite systems, $v_{x c}(\mathbf{r})$ decays asymptotically as $-1 / r[6]$, a result which has played an important role in the evolution of the energy functional construction. In contrast, little is known about $v_{x c}(\mathbf{r})$ near the nucleus. Inaccuracies in numerical calculations or unreasonable approximations made could even lead to divergent results at the nucleus, (see later discussion and Ref. [7].) It was recently proved that $v_{x c}(\mathbf{r})$ is finite at the nucleus. [8] Whether it behaves linearly or quadratically near the nucleus is not clear [9, 10]. In this paper it is pointed out that $v_{x c}(\mathbf{r})$ approaches the nucleus linearly, as shown in Eq. (2).

The asymptotic $-1 / r$ structure in the classically forbidden region is totally attributable to the Pauli correlation and it has been illustrated to arise from the Fermi hole [11]. The Coulomb correlation only makes a contribution to the next order of $O\left(1 / r^{4}\right)$ arising from the Coulomb hole [11]. It has been shown that the correlation-kinetic effects do make a contribution asympotically, but only to the order of $O\left(1 / r^{5}\right)[11]$. Hence in that region the correlation-kinetic effects are negligibly small. By contrast Eq. (2) indicates that, near the nucleus, the linear term of the spherically averaged $x c$ potential $\bar{v}_{x c}(r)$ arises entirely from the correlation-kinetic effects. (This understanding can also be reached via Quantal density functional theory (Q-DFT) [12]. In Ref. [12] it is explicitly shown that the Fermi-Coulomb hole makes no contribution to the linear term of $\bar{v}_{x c}(r)$.) We shall further argue that the linear term of $v_{x c}(\mathbf{r})$ is rather small, and $v_{x c}(\mathbf{r})$ is therefore nearly quadratic near the nucleus.

The understanding reached in this work should be useful in the construction of the KS system, especially via approximate $x c$ energy functionals. We shall demonstrate this with examples after the main derivations.

We start with deriving the following cusp relation for the density and the kinetic energy density at the nucleus for the interacting system,

$$
5 Z \bar{\rho}^{\prime \prime}(0)+\bar{\rho}^{\prime \prime \prime}(0)=\frac{8}{3} Z\left[4 Z^{2} \rho(0)+\bar{t}(0)\right]
$$

and the corresponding one for the KS system,

$$
\begin{aligned}
5 Z \bar{\rho}^{\prime \prime}(0)+\bar{\rho}^{\prime \prime \prime}(0)= & \frac{8}{3} Z\left[4 Z^{2} \rho(0)+\bar{t}_{s}(0)\right. \\
& \left.+\frac{3}{4 Z} \rho(0)\left(\bar{v}_{H}^{\prime}(0)+\bar{v}_{x c}^{\prime}(0)\right)\right],
\end{aligned}
$$


where the double and triple primes denote the second and third derivatives, respectively, with respect to $r$. To this end, we expand the ground state many-body wavefunction for the interacting $N$ electron system as

$$
\begin{aligned}
\Psi(\mathbf{r}, \mathbf{X}) & =\Psi(0, \mathbf{X})+a(\mathbf{X}) r+b(\mathbf{X}) r^{2}+c(\mathbf{X}) r^{3}+\ldots \\
& +\sum_{m=-1}^{1}\left[a_{1 m}(\mathbf{X}) r+b_{1 m}(\mathbf{X}) r^{2}\right] Y_{1 m}(\hat{r})+\ldots \\
& +\sum_{m=-2}^{2} b_{2 m}(\mathbf{X}) r^{2} Y_{2 m}(\hat{r})+\ldots
\end{aligned}
$$

for small $r$, where $\hat{r}=\mathbf{r} / r$, and $\mathbf{X}$ denotes $s, \mathbf{r}_{2} s_{2}, \ldots, \mathbf{r}_{N} s_{N}$. By substituting the wavefunction into the many-body Schrödinger equation, one obtains the following relations:

$$
\begin{aligned}
& a(\mathbf{X})+Z \Psi(0, \mathbf{X})=0 \\
& 2 b_{1 m}(\mathbf{X})+Z a_{1 m}(\mathbf{X})=0 \\
& 4 Z b(\mathbf{X})-Z^{3} \Psi(0, \mathbf{X})+6 c(\mathbf{X})=0
\end{aligned}
$$

These relations originate from the electron-nucleus cusp of the wavefunction, and were derived previously in Ref. [13]. With them, one can see that the density behaves as

$$
\begin{aligned}
\bar{\rho}(r)= & {\left[(1-Z r)^{2}+Z^{3} r^{3} / 3\right] \rho(0) } \\
& +2\left(r^{2}-5 Z r^{3} / 3\right) N \int d \mathbf{X} \operatorname{Re}\left[\Psi^{*}(0, \mathbf{X}) b(\mathbf{X})\right] \\
& +\left(r^{2}-Z r^{3}\right) N \int d \mathbf{X} \sum_{m=-1}^{1}\left|a_{1 m}(\mathbf{X})\right|^{2} / 4 \pi \\
& +O\left(r^{4}\right) .
\end{aligned}
$$

The kinetic energy density, $t(\mathbf{r})=\frac{1}{2} N \int d \mathbf{X} \nabla \Psi^{*}(\mathbf{r}, \mathbf{X}) \cdot \nabla \Psi(\mathbf{r}, \mathbf{X})$, behaves as

$$
\begin{aligned}
\bar{t}(r)= & \bar{t}(0)-\frac{2}{3} Z r\left[2 \bar{t}(0)-Z^{2} \rho(0)\right] \\
& -2 Z r N \int d \mathbf{X} \operatorname{Re}\left[\Psi^{*}(0, \mathbf{X}) b(\mathbf{X})\right]+O\left(r^{2}\right),
\end{aligned}
$$

where

$$
\bar{t}(0)=\frac{1}{2} Z^{2} \rho(0)+N \int d \mathbf{X} \sum_{m=-1}^{1} \frac{3}{8 \pi}\left|a_{1 m}(\mathbf{X})\right|^{2} .
$$

We note that the second term on the right hand side $(r h s)$ of the preceding expression for $\bar{t}(0)$ is absent in the literature [2]. Combining Eqs. (8) and (10) yields the relation of Eq. (41). 
Next we consider the corresponding KS system. We write $\phi_{n}(\mathbf{r})$ as

$$
\begin{aligned}
& \phi_{n}(\mathbf{r})=\sum_{l m} r^{l}\left[\quad A_{n l m}+B_{n l m} r+C_{n l m} r^{2}\right. \\
& \left.+D_{n l m} r^{3}+\ldots\right] Y_{l m}(\hat{r}) .
\end{aligned}
$$

Substituting Eq. (11) into Eq. (1) with $v_{\text {ext }}(\mathbf{r})=-Z / r$, one can obtain

$$
\begin{aligned}
& B_{n 00}+Z A_{n 00}=0, \\
& 2 B_{n 1 m}+Z A_{n 1 m}=0, \\
& 4 Z C_{n 00}-\left(Z^{3}+\bar{v}_{H}^{\prime}(0)+\bar{v}_{x c}^{\prime}(0)\right) A_{n 00}+6 D_{n 00}=0 .
\end{aligned}
$$

Evidently these relations similarly originate from the electron-nucleus cusp of the KS orbitals. Substituting Eq. (11) into the expression for the density for the KS system, $\rho(\mathbf{r})=\sum_{n=1}^{N}\left|\phi_{n}(\mathbf{r})\right|^{2}$, together with the relations in Eq. (12), one has

$$
\begin{aligned}
\bar{\rho}(r) & =\rho(0)\left[(1-Z r)^{2}+\frac{1}{3}\left(Z^{3}+\bar{v}_{H}^{\prime}(0)+\bar{v}_{x c}^{\prime}(0)\right) r^{3}\right] \\
& +\frac{1}{2 \pi} \sum_{n=1}^{N} \operatorname{Re}\left(A_{n 00}^{*} C_{n 00}\right)\left(r^{2}-\frac{5}{3} Z r^{3}\right) \\
& +\frac{1}{4 \pi}\left(r^{2}-Z r^{3}\right) \sum_{n=1}^{N} \sum_{m=-1}^{1}\left|A_{n 1 m}\right|^{2}+O\left(r^{4}\right) .
\end{aligned}
$$

Similarly, the kinetic energy density for the KS system, $t_{s}(\mathbf{r})=\frac{1}{2} \sum_{n=1}^{N} \nabla \phi_{n}^{*}(\mathbf{r}) \cdot \nabla \phi_{n}(\mathbf{r})$, behaves as

$$
\begin{aligned}
\bar{t}_{s}(r)= & \bar{t}_{s}(0)-\frac{2}{3} Z r\left[2 \bar{t}_{s}(0)-Z^{2} \rho(0)\right] \\
& -2 Z r \sum_{n=1}^{N} \frac{1}{4 \pi} \operatorname{Re}\left[A_{n 00}^{*} C_{n 00}\right]+O\left(r^{2}\right),
\end{aligned}
$$

where

$$
\bar{t}_{s}(0)=\frac{1}{2} Z^{2} \rho(0)+\frac{3}{8 \pi} \sum_{n=1}^{N} \sum_{m=-1}^{1}\left|A_{n 1 m}\right|^{2} .
$$

Combining Eqs. (13) and (15) leads to Eq. (15). Once again, we note that the second term on the rhs of Eq. (15) for $\bar{t}_{s}(0)$ is absent in the literature [2]. It is exactly this term and 
the corresponding term in $\bar{t}(0)$ on the rhs of Eq. (10) that make $\bar{t}(0) \neq \bar{t}_{s}(0)$, a fact which is critical to the nonzero linear term of $\bar{v}_{x c}(r)$. In fact, a comparison of Eq. (4) and Eq. (5) leads to $\bar{v}_{H}^{\prime}(0)+\bar{v}_{x c}^{\prime}(0)=4 Z\left[\bar{t}(0)-\bar{t}_{s}(0)\right] / 3 \rho(0)$, which equivalently implies Eq. (2), since $\bar{v}_{H}^{\prime}(0)=0$.

Only the properties of $\bar{t}(0)$ and $\bar{t}_{s}(0)$ have been employed in the preceding derivation, while accuracy to the linear terms in $\bar{t}(r)$ in Eq. (9) and $\bar{t}_{s}(r)$ in Eq. (14) enables us to obtain an interesting cusp relation,

$$
\bar{t}_{x c}^{\prime}(0)=-\frac{2}{3} Z \bar{t}_{x c}(0)
$$

with the aid of a comparision of the respective coefficients for the $r^{2}$ and $r^{3}$ terms in Eqs. (8) and (13).

To investigate further the near nucleus behavior of the KS exchange $v_{x}(\mathbf{r})$ and correlation $v_{c}(\mathbf{r})$ potentials separately, we employ the adiabatic coupling constant perturbation scheme [5]. In such a scheme, one has, instead of Eq. (2),

$$
\bar{v}_{x c}^{\lambda}(r)=\bar{v}_{x c}^{\lambda}(0)+\frac{4}{3} Z\left[\bar{t}^{\lambda}(0)-\bar{t}_{s}(0)\right] r / \rho(0)+O\left(r^{2}\right) .
$$

On the other hand, it has been shown that [14]

$$
v_{x c}^{\lambda}(\mathbf{r})=\lambda v_{x}(\mathbf{r})+v_{c}^{\lambda}(\mathbf{r})
$$

and $v_{c}^{\lambda}(\mathbf{r})$ commences in second order of $\lambda$. By comparing Eqs. (17) and (18), one arrives at Eq. (3). In the meanwhile one has

$$
\bar{t}_{x, c}^{\prime}(0)=-\frac{2}{3} Z \bar{t}_{x, c}(0)
$$

in addition to Eq. (16).

It is well known that $t_{x}(\mathbf{r})$ integrates to zero, i.e., $T_{x}=\int d \mathbf{r} t_{x}(\mathbf{r})=0$. Notice that $t_{x}(\mathbf{r})$ is however not necessarily zero except for homogeneous systems. Nevertheless, the fact that $T_{x}=0$ somewhat indicates that the linear term of $\bar{v}_{x c}(r)$ mainly arises from $\bar{v}_{c}(r)$, according to Eq. (3). In other words, the linear term is rather small, and $v_{x c}(\mathbf{r})$ is nearly quadratic near the nucleus. This argument might be corroborated by the fact that both the following sphericalized approximate exchange potentials approach the nucleus quadratically: (a) the Pauli correlated approximation $W_{x}(\mathbf{r})$ of Q-DFT [15], which is the part of the exchange potential arising purely from the Fermi hole,

$$
\bar{W}_{x}(r)=\bar{W}_{x}(0)+O\left(r^{2}\right) ;
$$


(b) the Krieger-Li-Iafrate (KLI) approximation [16] to the optimized exchange potential (OEP) [17],

$$
\bar{v}_{x}^{K L I}(r)=\bar{v}_{x}^{K L I}(0)+O\left(r^{2}\right)
$$

Equation (20) follows directly from the fact that the Fermi hole for an electron at the nucleus of a sphericalized system is spherically symmetric [12]. Equation (21) can be analytically derived. (It is worth mentioning that the exact OEP, being the exact exchange-only scheme [17], approaches the nucleus linearly according to the result of this paper.) Therefore, in contrast to the common wisdom that $v_{x}(\mathbf{r})$ dominates $v_{c}(\mathbf{r})$ as is the case in most other regions, $v_{c}(\mathbf{r})$ plays a much more significant role in the linear term of $v_{x c}(\mathbf{r})$ near the nucleus.

A nearly exact result for the density of the helium atom is available, which makes it an excellent testing ground for various approximate energy functionals. The single occupied KS orbital for the two electrons with opposite spins is simply $\phi(\mathbf{r})=\sqrt{\rho(\mathbf{r}) / 2}$. The kinetic energy density at the nucleus of the KS system can be readily shown, by employing this orbital, to be

$$
t_{s}(0)=\frac{1}{2} Z^{2} \rho(0)
$$

Therefore, according to Eq. (2), one has $v_{x c}^{\prime}(0)=2 Z\left[2 t(0) / \rho(0)-Z^{2}\right] / 3$. Further employment of the cusp relation of Eq. (44) yields

$$
v_{x c}^{\prime}(0)=\left[5 Z \rho^{\prime \prime}(0)+\rho^{\prime \prime \prime}(0)-12 Z^{3} \rho(0)\right] / 2 \rho(0)
$$

a result obtained previously in Ref. [10]. Furthermore, in this case, $v_{x}(r)=-v_{H}(r) / 2$. Therefore $v_{x}(r)$ has no contribution to the linear order, and $v_{c}(r)$ makes the entire contribution to the linear term of $v_{x c}(r)$. Notice that interestingly the second term on the rhs of Eq. (15) for $t_{s}(0)$ is absent in Eq. (22) in this special case, since both electrons occupy the $1 s$ orbital. This is in fact also true for three- or four-electron systems like Li and Be atoms and their isoelectronic sequences since all electrons occupy single particle states with $l=0$. The corresponding second term on the rhs of Eq. (10) for $t(0)$ is however nonzero, which makes the entire contribution to $v_{x c}^{\prime}(0)$.

\section{Table 1}

Comparison of the exact results for $v_{x, c}(0)$ and $v_{x, c}^{\prime}(0)$ and those calculated with LDAs for the helium atom. (a), (b) and (c) respectively refer to Vosko-Wilk-Nusair, Perdew-Wang, 
and Wigner parametrizations [19] for the correlation energy functionals. Atomic units are

\begin{tabular}{ccccc}
\multicolumn{5}{c}{ used. } \\
\hline \hline & $v_{x}(0)$ & $v_{c}(0)$ & $v_{x}^{\prime}(0)$ & $v_{c}^{\prime}(0)$ \\
\hline Exact & -1.69 & -0.062 & 0 & 3.8 \\
\hline \multirow{5}{*}{ LDA } & -1.51 & $-0.092(\mathrm{a})$ & & $0.036(\mathrm{a})$ \\
& & $-0.082(\mathrm{~b})$ & 2.02 & $0.035(\mathrm{~b})$ \\
& & & $0.0025(\mathrm{c})$ \\
\hline
\end{tabular}

All the commonly used generalized gradient approximation (GGA) functionals suffer from spurious divergence for $v_{x c}(\mathbf{r})$ at the nucleus [7, 10]. This is also true for the later proposed GGA in Ref. [18]. The divergence originates from terms in $v_{x c}(\mathbf{r})$ containing factor $\nabla^{2} \rho(\mathbf{r})$ which has behavior of $O(1 / r)$ at the nucleus. The local density approximation (LDA), on the other hand, yields reasonable result for $v_{x}(0)$ and $v_{c}(0)$ as shown in Table 1 for the helium atom. But, as shown in Table 1, the LDA for the exchange component largely overestimates $v_{x}^{\prime}(0)$, and the three LDAs proposed for the correlation component all hugely underestimate $v_{c}^{\prime}(0)$, against the fact revealed in this paper that the correlation component plays a dominant role in the linear term of $v_{x c}(\mathbf{r})$ near the nucleus.

Finally we take the electrostatic interpretation of the KS $x c$ potential [20] as another example to illustrate the possible implications of our investigation for the construction of the KS system. In this interpretation, the concept of static exchange-correlation charge density $q_{x c}(\mathbf{r})$ is introduced for the KS xc potential,

$$
\nabla^{2} v_{x c}(\mathbf{r})=-4 \pi q_{x c}(\mathbf{r})
$$

The result shown in Eq. (2) indicates that $q_{x c}(\mathbf{r})$ diverges at the nucleus,

$$
\bar{q}_{x c}(r \rightarrow 0)=-\frac{2}{3 \pi r} Z\left[\bar{t}(0)-\bar{t}_{s}(0)\right] / \rho(0) .
$$

This unphysical feature reveals inherent shortcomings of the electrostatic interpretation of $v_{x c}(\mathbf{r})$. It implies that the part of $v_{x c}(\mathbf{r})$ due to the correlation-kinetic effects can not be properly interpreted in terms of a static charge density. Furthermore, according to the result in Eq. (3), the shortcomings persist in the analogous interpretation [20] of the exchange $v_{x}(\mathbf{r})$ and correlation $v_{c}(\mathbf{r})$ potentials, separately, in terms of static exchange $q_{x}(\mathbf{r})$ and correlation $q_{c}(\mathbf{r})$ charge densities. 
It might be necessary to point out the following fact before we close the paper. As mentioned in the introduction, in addition to the traditional exchange-correlation energy $E_{x c}[\rho]$ of the conventional many-body theory, which arises purely from the interaction between an electron and its Fermi-Colomb hole, there is a contribution of the correlation-kinetic energy $T_{x c}[\rho]=\int d \mathbf{r}\left[t(\mathbf{r})-t_{s}(\mathbf{r})\right]$ to the KS exchange-correlation energy: $E_{x c}^{K S}[\rho]=E_{x c}[\rho]+T_{x c}[\rho]$. Correspondingly, $v_{x c}(\mathbf{r})=\delta E_{x c}[\rho] / \delta \rho+\delta T_{x c}[\rho] / \delta \rho$. The linear term in $\bar{v}_{x c}(r)$ in Eq. (22) is identified as the correlation-kinetic effects, but it remains not clear whether it arises entirely from $\delta T_{x c}[\rho] / \delta \rho$. Nevertheless, the result in this work indicates that, near the nucleus, $T_{x c}[\rho]$ plays a significant role in the performance of the approximate KS $x c$ energy functionals.

The author is grateful to Prof. V. Sahni for discussions and help, and Prof. C. J. Umrigar for providing accurate data on the density of the helium atom. This work was supported by the Chinese National Science Foundation under Grant No. 10474001.

[1] P. Hohenberg and W. Kohn, Phys. Rev. 136, B864 (1964); W. Kohn and L. J. Sham, ibid. 140, A1133 (1965).

[2] R. M. Dreizler and E. K. U. Gross, Density Functional Theory, (Springer-Verlag, Berlin, 1990).

[3] R. G. Parr and W. Yang, Density Functional Theory of Atoms and Molecules, (Oxford University Press, New York, 1989).

[4] Here we mean the traditional Fermi-Coulomb hole in many-body theory, in contrast to the exchange-correlation hole extensively used in HKS-DFT. The exchange-correlation hole is the coupling constant average of the Fermi-Coulomb hole in the adiabatic coupling constant scheme, (see Ref. [5] and the later discussion in the present paper.)

[5] J. Harris and R. O. Jones, J. Phys. F 4, 1170 (1974); D. C. Langreth and J. P. Perdew, Solid State Commun. 17, 1425 (1975); O. Gunnarsson and B. Lundqvist, Phys. Rev. B 13, 4274 (1976).

[6] C.-O. Almbladh and U. von Barth, Phys. Rev. B 31, 3231 (1985); L. J. Sham, ibid. 32, 3876 (1985).

[7] C.-O. Almbladh and A. C. Pedroza, Phys. Rev. A 29, 2322 (1984); A. C. Pedroza, ibid. 33, 804 (1986); D. C. Langreth and M. J. Mehl, Phys. Rev. B 28, 1809 (1983); J. P. Perdew and Y. Wang, ibid. 33, 8800 (1986); A. D. Becke, Phys. Rev. A 38, 3098 (1988); J. Chen et al., 
Phil. Mag. B 69, 1001 (1994).

[8] Z. Qian and V. Sahni, Int. J. Quantum Chem. 79, 205 (2000); X. Y. Pan and V. Sahni, Phys. Rev. A 67, 012501 (2003).

[9] D. W. Smith et al., Int. J. Quantum Chem. Symp. 13, 103 (1979); E. R. Davidson, Int. J. Quantum Chem. 37, 811 (1990); A. Nagy and K. D. Sen, J. Chem. Phys. 115, 6300 (2001); K. Peirs, D. Van Neck, and M. Waroquier, Phys. Rev. A 67, 012505 (2003).

[10] C. J. Umrigar and X. Gonze, Phys. Rev. A 50, 3827 (1994); C. Filippi et al., in Recent Developments and Applications of Density Functional Theory, edited by J. M. Seminario, (Elsevier, Amsterdam, 1996).

[11] Z. Qian and V. Sahni, Int. J. Quantum Chem. 70, 671 (1998); Phys. Rev. A 57, 4041 (1998).

[12] Z. Qian and V. Sahni, Phys. Rev. A (accepted).

[13] T. Kato, Commun. Pure. Appl. Math. 10, 151 (1957); W. A. Bingel, Z. Naturforsch. A 18a, 1249 (1963); R. T. Pack and W. B. Brown, J. Chem. Phys. 45, 556 (1966); V. A. Rassolov and D. M. Chipman, ibid. 104, 9908 (1996).

[14] A. Görling and M. Levy, Phys. Rev. B 47, 13105 (1993).

[15] M. K. Harbola and V. Sahni, Phys. Rev. Lett. 62, 489 (1989); V. Sahni, Quantal Density Functional Theory, (Springer-Verlag, Berlin, Heidelberg, 2004).

[16] J. B. Krieger et al., Phys. Rev. A 45, 101 (1992).

[17] R. T. Sharp and G. K. Horton, Phys. Rev. 90, 317 (1953); J. D. Talman and W. F. Shadwick, Phys. Rev. A 14, 36 (1976); V. R. Shaginyan, ibid. 47, 1507 (1993).

[18] J. P. Perdew et al., Phys. Rev. Lett. 77, 3865 (1996).

[19] S. H. Vosko ibid., Can. J. Phys. 58, 1200 (1980); J. P. Perdew and Y. Wang, Phys. Rev. B 45, 13244 (1992); E. P. Wigner, Phys. Rev. 46, 1002 (1934), (see also G. D. Mahan, Many-Particle Physics (Plenum, New York, 1981).)

[20] S. Liu et al., J. Chem. Phys. 111, 6197 (1999); A. Görling, Phys. Rev. Lett. 83, 5459 (1999); P. W. Ayers and M. Levy, J. Chem. Phys. 115, 4438 (2001); N. H. March, Phys. Rev. A 65, 034501 (2002); K. D. Sen et al., J. Chem. Phys. 117, 4684 (2002). K. D. Sen, F. J. Luque, Int. J. Quantum Chem. 101, 231 (2005). 